\title{
Certain Tendencies in the Development of the Method to Assess Group Political Attitudes
}

\author{
Ivan Nikolaevich Mochtchenko \\ Mariya Ivanovna Ivanova \\ Mikhail Dmitrievich Rozin \\ Valeriy Petrovich Svechkarev
}

Southern Federal University, Russia

Doi:10.5901/mjss.2015.v6n3s2p73

\begin{abstract}
The results obtained by evaluating socio-political tension are used for the practical optimization of the life spheres of today's society. That is why it seems very topical to carry out research aimed at the development of methods how to analyze and evaluate various aspects of socio-political tension including those formed by integrating methods from different scientific fields. This paper is aimed at a theoretical test of method, a practical test of measuring model and their concerted integration with the mathematical methods and models for the interpretation of research results concerning socio-political tension. The theoretical and practical tests of the suggested method confirm the principal possibility of quantitative assessment of socio-political tension. The research included the test of an integral analysis of tendencies in group political attitude on the basis of the developed method. This method studies emotional, cognitive and behavioral components. This became possible thanks to the concerted integration of socio-psychological research principles, projective techniques and mathematical modeling. The authors propose to call this method a psycho-semantic phenomenological modeling of group political tension.
\end{abstract}

Keywords: Socio-political tension; social attitude; semantic differential; ideal point model.

\section{Introduction}

The evaluation of socio-political tension is used for the practical optimization of the main life spheres of today's society. That is why it seems urgent to carry out research aimed at the development of methods for the analysis and assessment of various aspects concerning socio-political tension including those formed by integrating methods from different scientific fields (Dobrenkov and Kravchenko, 2006). It is well-known that while studying social reality one should first of all study the "social attitude". The study of social attitude is always connected with the problem of method and boils down to the development of its measurement model (Krupenkova, 2002). This research can be focused on the behavioral component (of an individual or a group) of the attitude to one or another social object. In this case, this is a political order. The behavioral component is determined to a large extent by the cognitive and affective components of these attitudes (Tsiptsuk, 1984), i.e. by the perception of political order. Formally, this follows from the paradigm of attitude's threecomponent structure which includes cognitive, emotional and behavioral components (Smith, 1954). In this case, behaviour towards the object of attitude is determined by the awareness and assessment of social attitude object at the level of liking or dislike. If one manages to reconcile the components, the attitude will perform a regulatory function. Otherwise, the uncontrolled behaviour of an individual or a group is of high probability. Thus the efficiency of research in the field of socio-political tension is connected with the development of models to measure the mentioned perception parameters which influence the behavioral component.

Projective techniques and models are the most widespread in this field (Sokolova, 1980; Frank, 2000). Projective techniques are aimed at measuring personality traits and forecasting social behaviour (Korner, 2000). They have a number of peculiarities due to which they differ significantly from standard methods. Particularly, an essential condition for the principle of projection is the ambiguity and indefiniteness of stimulus material (Lazarus, 2000). During the interaction with stimulus material, personality projects its inner world: aspirations, anxiety and appraisals. These methods are principally characterized by a qualitative approach to the study of personality. That is why the usage of projective techniques to study socio-political tension needs a theoretical test of these methods, a practical test of measurement 
models and a concerted integration with mathematical methods and models for the interpretation of research results. This is the goal of this paper. The paper contains a method worked out by the author. It helps to measure perception parameters which influence the behavioral component. This method includes the principles of socio-psychological research of projective techniques and mathematical modeling methods (Rozin, 2012). The authors propose to call this method a psycho-semantic phenomenological modeling (Moschenko, 2010).

\section{Method: Semantic Differential}

This paper contains a theoretical and practical test of the method. The test is carried out during the analysis of the cognitive and emotional components of group attitudes. It is based on experimental questionnaire among the students of Rostov State Construction University (RSCU) (RSCU, 2014). In the period of 2009-2013, 770 students of RSCU took part in the questionnaire: 48\% men and 52\% women. They were aged 16 to 21 years: 18 years - 57\%; 19 years - 22\%; 17 years $-15 \%$, and others. By nationality, $85 \%$ were Russian; $4.8 \%$ were Armenians, and others.

The emotional component of group political attitude was measured by the method of semantic differential (Osgood, 1952). The cognitive and behavioral components of political attitude was evaluated by direct questioning (Dobrenkov and Kravchenko, 2006). Semantic differential is a questioning method (Osgood et al., 1957). The respondents are suggested to estimate a social process on 20 seven-point scales (from -3 to +3 ). Each of them is formed by a pair of antonymous adjectives. As a result, individual and group semantic spaces are built. The assessments of an object act as its coordinates (Osgood et al., 1972). Some of these adjectives ("good - bad", "kind - evil") can characterize a studied social phenomenon by their denotative (objective) meaning. The scales formed by them reflect how the respondents interpret the social phenomenon at the cognitive level. Another part of pair adjectives ("men's - women's", "tasty - tasteless") can be used to characterize a social process only in an allegoric sense because of their connotative (personal, subjective) content (Osgood et al., 1957). It is this kind of scales that an unconscious affective (emotional) component of perception is projected. In the method of semantic differential, the perception of some object or process is modeled by an image in an abstract semantic 20-dimentional space. The decision what component of perception to study (cognitive or unconscious) depends on where the scale-making adjectives are shifted relative to the object: in denotative or connotative direction.

The cognitive scales can be interpreted by their denotative meaning. But the scales of unconscious affective perception are hard to interpret directly because here there is a purely personal content. At this stage, a problem appears what scale to choose to measure a social phenomenon. The more measurement is shifted to the cognitive component, the easier it is to interpret the obtained image of an object in semantic space. However, the specificity of the semantic differential is that it allows measuring just the deep unconscious component of perception. This component is less susceptible to external influence and is more connected with resultant behaviour. This is the reason why the adjectives in the main part of scales in our questionnaire are used by their connotative meaning. In this case, the semantic space acts as an abstract multidimensional space without any connection with reality. Besides, it is difficult to make a conclusion only by one object. If we include at least one more object in the research, new usable characteristics will appear in the abstract space.

\section{Method: Development and Addition}

As it is shown in (Coombs et al., 1954; Coombs, 1957), while assessing several objects by answers, one should take into account how the respondents interpret these objects. In this case, the most adequate model is the model of ideal point. According to this model, respondents have their own idea about an ideal object from the analyzed group. The closer the real object is to the ideal one, the oftener a respondent prefers it in his answer. Hence, it appears that the similarity of images of two objects in semantic space characterizes the similarity of perception of these objects by respondents. In our method, we used not one but two ideal points: positive and negative. Respondents were to assess a real object and two ideal constructs using the method of semantic differential. When using two ideal points, we specify the direction and scale in the semantic space to evaluate the perception of real constructs. We detected the similarity of images in 20dimensional semantic space by relative Cartesian distance. The relative distance between the images of ideal constructs was equal to two.

So, to evaluate the perception of real objects, we used perception level $r$, normalized from -1 (the perception of a negative ideal construct) to +1 (the perception of a positive ideal construct). It is possible to analyze the degree of similarity or difference of integral perception by the relative position of these images without opening the inner structure of this perception. This is what characterizes the phenomenological approach to the interpretation of data obtained in 
research. The phenomenological approach in science is an approach which helps to build a theory for observed phenomena and their description without studying inner processes. This integral approach is quite enough when research is aimed at detecting perception parameters which influence the behaviour. The analysis of inner structure is not in the subject of this research.

We have the following goal. We need to find and measure some object perception characteristic which determines the behavioral component. Moreover, it is necessary to ascertain whether respondents will support or deprecate this social object. We use ideal constructs to specify such a characteristic. They are formulated not only as ideally positive or ideally negative objects but with behavioral orientation: ideally positive, fully supported by the respondent, and ideally negative, fully rejected (down to an armed resistance). The introduced level characterized by the degree of closeness of real object perception to the perception of ideal constructs will give the sought-for characteristic which influences the behaviour of respondents. It means that the purely phenomenological approach begins just when ideal constructs are chosen. This approach is the whole complex.

It should be noted that that real perception initially specifies the position of political order images in semantic coordinates. Besides, it determines relative distances from the images of real constructs (in our case, political) to the images of ideal constructs (positive and negative). Using Coombs' method, we can show that one should consider such dependences monotonous while solving an inverse problem: to find the level of perception by relative distances.

In linear approximation, with a glance to the above normalization, we find that the perception level of real order is equal to the half-difference of respective relative distances:

$$
r_{x}=\left(d_{-1 x}-d_{+1 x}\right) / 2,
$$

where ${ }^{d_{+1 x}}$ is the relative distance from the image of real order to the image of ideally positive order; ${ }^{d-1 x}$ is the distance to the image of negative construct.

\section{Findings}

On the basis of obtained data, we built averaged semantic descriptions for all studied types of political orders (Figure 1). Here we use the following code to designate the type of studied political orders: 0.010 - real local political order; -0.010 real central political order; 1 - ideal positive order; -1 - ideal negative order. It should be noted that only one real political order was measured in 2009. That is why the semantic description of the real construct is in lines 0.010 and -0.010 .

Then we calculated the levels of emotional perception of real orders. Table 1 and Figure 2 show the results.

Table 1. The levels of emotional perception of political order calculated in linear approximation

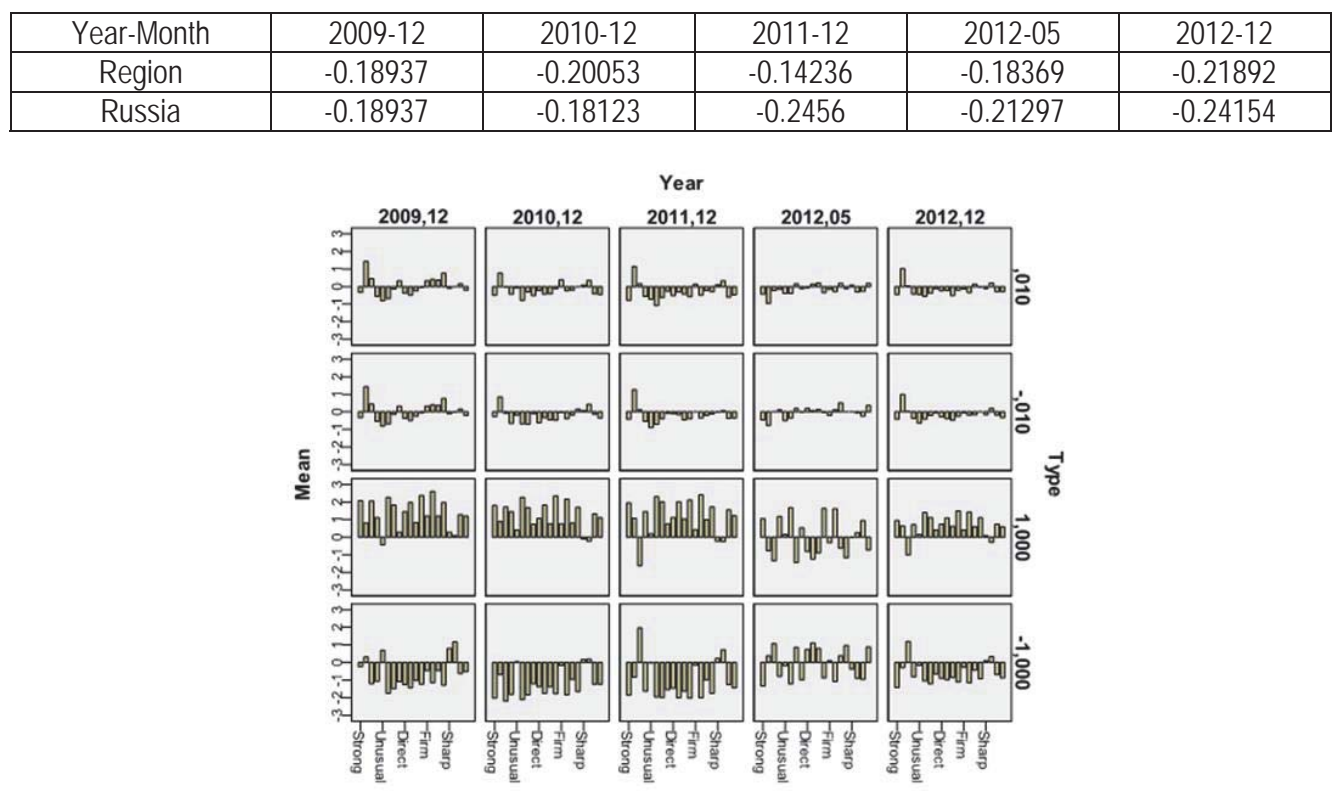

Figure 1. The levels of emotional perception of political order calculated in linear approximation. The vertical line is the levels of perception. The horizontal line is the year and month of research. 


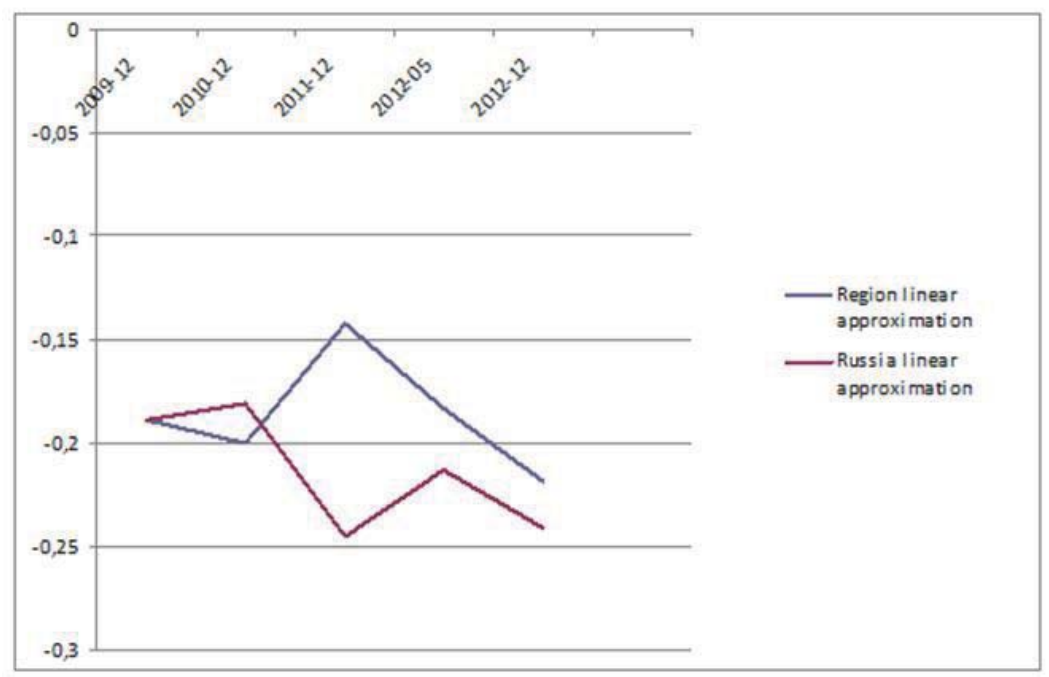

Figure 2. The levels of emotional perception of political order calculated in linear approximation. The vertical line is the levels of perception. The horizontal line is the year and month of research.

From 2009 till the end of 2012, there was a downward trend of perception level both in the region and in Russia (with a positive leap to in 2011 in the region).

For more detailed and adequate results, the perception levels of political orders were calculated in nonlinear approximation. The degree of necessary nonlinearity was found in terms of the conception of model typicalness using the results of the catastrophe theory (Poston and Stewart, 1978). Following the phenomenological approach, we supposed that the speed the perception level changes with is found by some unknown function $\mathrm{F}$ which depends mainly on the level of perception and introduced relative distances. Due to the unidimensionality of perception level $r$, unknown function $\mathrm{F}$ can be replaced by the negative gradient of another unknown function $F$ named a potential of perception:

$$
d r / d t=-d F / d r^{\prime}
$$

The potential of perception depends on the same parameters as F. These parameters are always preset with a certain error and have a stochastic nature. Hence, $F$ is some function of general position (in mathematical sense).

As the catastrophe theory shows (Poston and Stewart, 1978), the form of a typical potential is determined only by the dimensionality of order's parameter (in our case, perception level $r$ ) and external parameters (relative distances introduced above). For our problem:

$$
F=(1 / 4) r^{\prime} 4-(1 / 2) a r^{\prime} 2-b r^{\prime},
$$

where phenomenological parameters $a, b$ and $r^{\prime}$ near the studied point are connected with the above mentioned relative distances and the sought-for perception level $r$ by linear dependences:

$$
a=\left(d_{+1 x}+d_{-1 x}\right) / 2 ; b=\left(d_{-1 x}-d_{+1 x}\right) / 2 ; r^{\prime}=m r .
$$

Scale factor $m$ is found by calibration points (ideal constructs with known perception levels).

Potential (2) and equation (1) fully allow solving a statistical problem of finding the level of perception by the results of the questionnaire. Besides, they allow modeling the change of perception level. Data obtained with the help of nonlinear approximation (Table 2 and Figure 3 ) confirm the above conclusions about the downward trend in the emotional perception of local and central authorities by the end of 2012.

Table 2. The levels of emotional perception of political order calculated in nonlinear approximation.

\begin{tabular}{|c|c|c|c|c|c|}
\hline Year-Month & $2009-12$ & $2010-12$ & $2011-12$ & $2012-05$ & $2012-12$ \\
\hline Region & -0.24594 & -0.24594 & -0.19697 & -0.25159 & -0.29206 \\
\hline Russia & -0.24594 & -0.27688 & -0.32981 & -0.28536 & -0.32026 \\
\hline
\end{tabular}




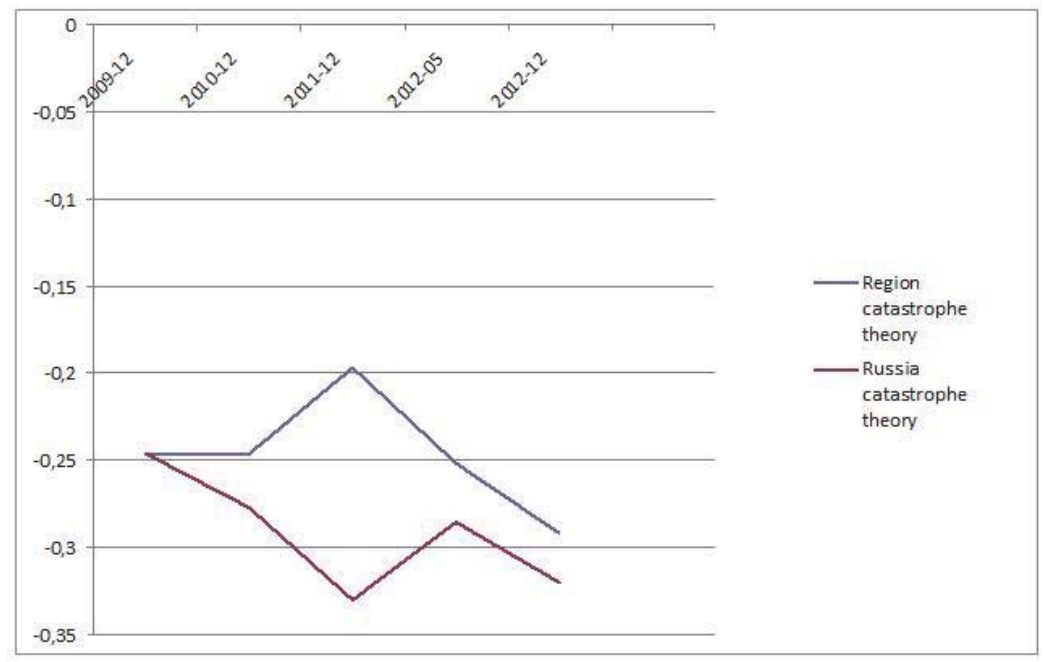

Figure 3. The levels of emotional perception of political order calculated in nonlinear approximation. The vertical line is perception levels; the horizontal line is the year and month of research.

\section{The Discussion of Results Obtained by Measuring the Cognitive Component of Political Attitudes}

The cognitive and behavioral components of political attitudes were measured by direct survey simultaneously with the method of semantic differential in order to study the reasons of this downward trend and to test the proposed method.

The questionnaires developed for the research were modified and supplemented from year to year. In 2012, the questionnaire was enlarged and new material appeared. It became possible to measure the cognitive and behavioral components of group political behaviour. To measure the cognitive component, respondents were suggested to evaluate the political situation in Rostov Region (hereinafter, the Region) and Russia in general, the fairness of applied policy and to express their approval or disapproval of decisions made by authorities. This range of problems underwent factor analysis (Tolstova, 2000; Nasledov, 2005): for the Region and Russia separately. For every case, we found one factor describing the $100 \%$ dispersion of initial results. This very factor was taken as a level of cognitive perception of political order (Table 3).

Table 3. The matrix of factor loading for the cognitive level of political order perception

\begin{tabular}{|c|c|c|c|}
\hline Approval of decisions & Policy fairness & The evaluation of political situation & \\
\hline 0.8822781 & 0.956586 & 0.682336 & In the Region \\
\hline 0.848106 & 0.994284 & 0.93164 & In Russia \\
\hline
\end{tabular}

Then, after the normalization from -1 to +1 , the levels of cognitive component were calculated for the Region and Russia in general. In so doing, -1 means the absolutely negative level of cognitive perception, and +1 means the absolutely positive level. The levels were calculated in linear approximation by average values. Table 4 shows the data obtained.

Table 4. The levels of cognitive perception of political order.

\begin{tabular}{|c|c|c|}
\hline Year & In Russia & In the Region \\
\hline $2012-12$ & -0.27 & -0.23 \\
\hline $2013-03$ & -0.23 & -0.20 \\
\hline
\end{tabular}

Both at the end of 2012 and in the middle of 2013, the cognitive perception level of both territories is insignificantly negative. At the same time, in both cases, the level of perception grows during six months, but remains in the negative zone. Cognitive perception reveals that respondents think better of the political situation in the Region than in Russia in general.

In the applied questionnaire, in 2009-2013, there were also two questions which characterize the deprivational 
component. Group tension appears when the group begins to notice the gap between its aspirations and available means. So, when the level of group relative deprivation increases (Myers, 1997), it can point the increase of tension, including the political one, on the group under study. The subjective opinion of respondents about the level of economic wellbeing and social security evaluates the level of deprivation.

The respondents were suggested to assess the level of their economic wellbeing and social security from "a very low level" to "a very high level". We factorized the data (Tolstova, 2000; Nasledov, 2005). It appeared that the indications of economic wellbeing and social security reveal one factor which describes the $100 \%$ dispersion of initial results (Table 5).

Table 5. The matrix of factor loading for the level of relative deprivation

\begin{tabular}{|c|c|}
\hline Economic wellbeing & Social security \\
\hline 0.753128 & 0.81891 \\
\hline
\end{tabular}

For further analysis, we performed normalization from 0 to 1 , where 0 is the absolute absence of deprivation, and 1 is the highest level of relative deprivation. Figure 4 shows the results. The vertical axis is the averaged level of deprivation; the horizontal axis is the year of survey. In 2009-2010, there was a jump from 0.348 to 0.522 . Then the relative deprivation remained at the stable middle level tile end of 2012. In mid-2013, the level of relative deprivation rose slightly to 0.555 .

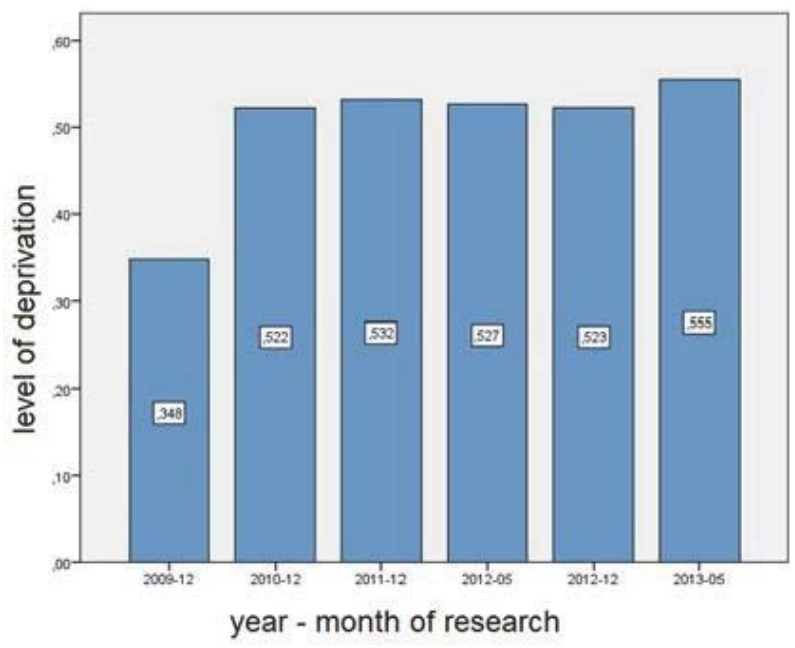

Figure 4. The distribution diagram for deprivation levels by the year of research

\section{The Discussion of Results Obtained by Measuring the Behavioral Component of Political Attitudes}

The behavioral component of group attitude is also found by direct survey. The questionnaire contained seven questions. The respondents were suggested to assess real and possible political actions or their character: the assessment of the involvement in political events; the participation in election or other social movements; emotional involvement in politics; the discussion of political news in family and among friends; the interest in these news and the attitude to mass actions. The analysis of different combinations of seven indications allowed dividing these indications into two groups and finding one factor for each of them (Tatarova, 1999). This factor was to describe the $100 \%$ dispersion of its initial data (Table 6). By its meaning, the first factor characterizes a behavioral action (actual behavior). In Table 6, it is designated " $F$-pov_act". The second factor describes the valuation level of behavioral component of group attitude (i.e. the perception of behavior or the attitude to behavior). In Table 6, this factor is designated "F-pov_ment". By these factors, the levels of active and valuation behavioral component were calculated in linear approximation. For behavioral activity, we used normalization from -1 to +1 , where -1 is the full absence of behavioral component, and +1 is the highest degree of activity. For the valuation level of behavioral component, +1 is a positive valuation, and -1 is a negative behavioral valuation (Table 7 ). 
Table 6. The matrix of factor loading for the behavioral component by two factors.

\begin{tabular}{|c|c|c|c|c|}
\hline & Participation & Voting & Participation in social movements & \\
\hline F_pov_act & 0.59007 & -0.51645 & -0.35884 & \\
\hline & Involvement & Discussion & Attitude to protests & Interest in news \\
\hline F_pov_ment & 0.902883 & 0.809663 & 0.602298 & 0.757749 \\
\hline
\end{tabular}

Table 7. The levels of cognitive perception of political order

\begin{tabular}{|c|c|c|}
\hline Year & The active level of behavior & The valuation level of behaviour \\
\hline $2012-12$ & -0.248 & 0.027 \\
\hline $2013-03$ & -0.268 & -0.096 \\
\hline
\end{tabular}

Then we performed the second factorization using obtained factors. The factor analysis by these levels showed that it is possible to introduce one factor which describes the general level of behavioral component of group attitude (Table 8).

Table 8. The matrix of factor loading for the general behavioral component

\begin{tabular}{|c|c|}
\hline F_pov_act & 0.544282 \\
\hline F_pov_ment & 0.616508 \\
\hline
\end{tabular}

For this analysis, we used normalization similar to the previous analysis: from -1 to +1 , where -1 is the full absence of integral behavioral component, and +1 is the highest degree of behavior (Figure 5). The levels of behavioral component of group attitude were also calculated in linear approximation by average values. For the end of 2012, the general level of behavioral component is -0.052 , and for mid-2013, this level is -0.148 .

As the results show, in the end of 2012, the behavioral component reveals the neutral level close to zero. This indicates a certain political detachment. By the middle of 2013 , there is a downward tendency to the insignificant level.

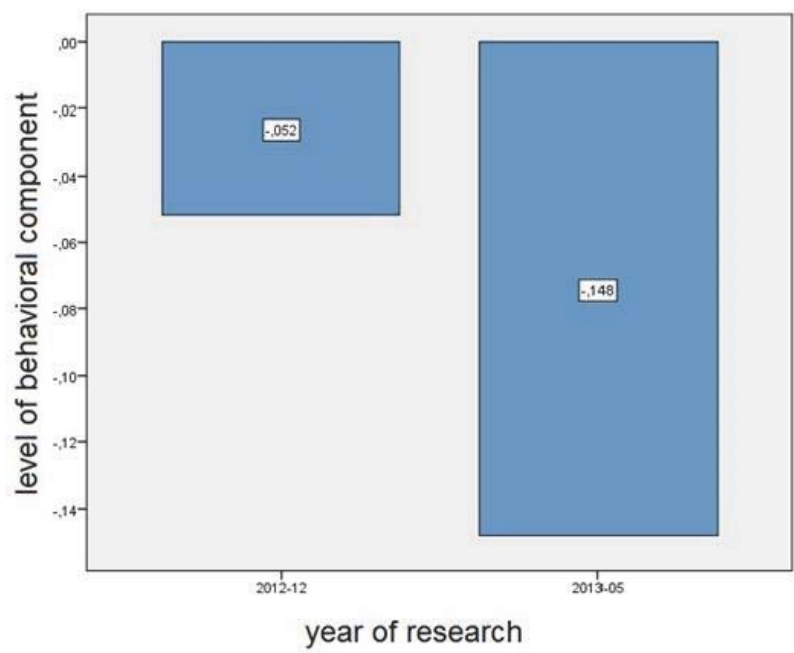

Figure 5. The diagram of behavioral component by the year of research

\section{Conclusion}

In conclusion, we would like to note that, besides the questions revealing the cognitive and behavior components, the developed questionnaire included a question for how young people appraise their life prospects. We considered this question earlier as the component of the relative deprivation level, but then we analyzed it separately because the factor analysis of initial data showed that the level of prospects is independent and is not included in other factors. When asked "Please, appraise your life prospects", respondents give very positive answers (Figure 6, Table 9). This is determined by the characteristics of the audience: students believe in their good future and make generally optimistic plans. 


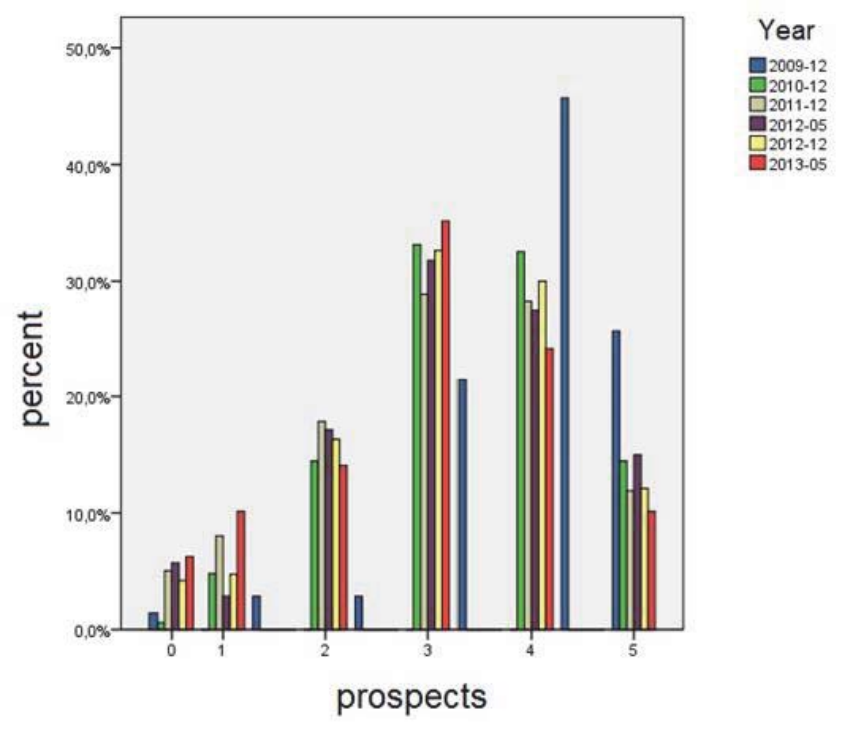

Figure 6. The distribution diagram for the appraisal of one's prospects by the year of research

Table 9. The average values of life prospects level by the year of research

\begin{tabular}{|c|c|}
\hline \multicolumn{2}{|c|}{ Average } \\
\hline Year & Prospect \\
\hline $2009-12$ & 4.02 \\
\hline $2010-12$ & 3.36 \\
\hline $2011-12$ & 3.03 \\
\hline $2012-05$ & 3.18 \\
\hline $2012-12$ & 3.16 \\
\hline $2013-05$ & 2.91 \\
\hline Total & 3.15 \\
\hline
\end{tabular}

The obtained results show the correlation between the level of relative deprivation (Figure 4) and the level of life prospects (Table 9). Besides, in 2009-2010 there is a decrease from 4.02 to 3.36 by average values. Gradually, year by year, the appraisal of life prospects decreases and reaches 2.91 in 2013 by average values. But, in spite of the decrease, students continue to appraise their future positively and above average.

One of the factors that determine political tension is a high level of relative deprivation. As it is stated above, the level of deprivation made a big jump in 2009. This corresponds to the period of crisis in the country. Besides, there is a slight decrease in 2013 but its small value cannot cause such drop of the emotional perception of local and central authorities.

By the example of the analysis of group political attitudes, we showed that the data for the affective and cognitive components do not correlate with each other. However, the final behavioral component cannot be described by direct correlation. It reflects deep inner processes inside an individual and a group. So, we can conclude that the threedimensional (what I know, what I feel and what I do) consideration of group attitudes cannot come to nothing more than a correlation between its components. The problem remains unsolved and requires further study.

The theoretical and practical tests of the proposed method performed in the paper confirm a possibility in principle to find a quantitative evaluation for socio-political tension. In the research, we tested the integral analysis of tendencies in group political attitudes on the basis of the developed method which includes the study of the emotional, cognitive and behavioral components. It became possible thanks to the concerted integration of socio-psychological research principles, projective techniques and mathematical modeling. The authors propose to call this method a psycho-semantic phenomenological modeling of group political tension. 


\section{Acknowledgements}

The authors note with gratitude that this paper was carried out within the framework of the state assignment given by the Ministry of Education and Science of the Russian Federation No. 014/174, project code 2119.

\section{References}

Dobrenkov, V.I. \& Kravchenko, A.I. (2006). The Methods for Sociological Research. Moscow: Infra-M.

Korner, A.F. (2000). The Theoretical Study of Capacity Limits of Projective Techniques. Projective Psychology. Moscow: April Press, EKSMO-Press.

Krupenkova, N.V. (2002). The Models of Interaction between Social Attitude and Behavior. Sociological Expariments. Collection of Scientific Articles. Moscow: RAS.

Lazarus, R.S. (2000). Definiteness and Indefiniteness in Projective Techniques. Projective Psychology. Moscow: April Press, EKSMOPress.

Myers, D. (1997). Social Psychology. Saint-Petersburg: Piter.

Moschenko, I.N. (2010). The Psycho-semantic Phenomenological Model of Group Political Tension. The Don Engineer Review: 1. from http://www.ivdon.ru/magazine/archive/n1e2010/173/

Nasledov, A.D. (2005). SPSS: Computer-Aided Data Analysis in Psychology and Social Sciences. Saint-Petersburg: Piter.

Osgood, C., Suci, G. \& Tannenbaum, P. (1972). The Semantic Differential Method in Aesthetic Research and Related Problems. Semiotics and Art Measuring. Moscow: Mir.

Rozin, M.D., Moschenko I.N. \& Ivanova, M.I. (2012). The Integration of Sociological and Mathematical Methods in Monitoring Group Attitudes. The Scientific Heritage of Yu.A. Zhdanov and Contemporary Problems in Modeling Complex Sociological Systems (as exemplified in South Russia): International Scientific Readings. Rostov-on-Don.

The Rostov State Construction University: about the University. Retrieved September 30, 2014 from http://www.rgsu.ru/university lindex.php

Sokolova, E.T. (1980). The Projective Techniques in the Study of Personality. Moscow: MSU.

Tatarova, G.G. (1999). The Methodology of Data Analysis in Sociology (Introduction). Moscow: NOTA BENE.

Tolstova, Y.N. (2000). The Analysis of Sociological Data: Methodology, Descriptive Statistics, the Study of Links between Nominal Indications. Moscow: Nauchny Mir.

Tsiptsuk, V.G. (1984). On the Correspondence between the Emotional-Cognitive and Behavioral Components of Social Attitude. The Points of the Anniversary Scientific Conference.

Frank, L.K. (2000). Projective Techniques in Personality Study. Projective Psychology. Moscow: April Press, EKSMO-Press.

Coombs, C.H. (1975). A Note on the Relation between the Vector Model and the Unfolding Model for Preferences. Psychometrika, 40(1), 115-116.

Coombs, C.H., Raiffa, H. \& Thrall, R.M. (1954). Some views on mathematical models and measurement theory. Psychological Review, $61,132-144$.

Osgood, C.E., Suci, G.J. \& Tannenbaum, P.H. (1957). The Measurement of Meaning: Monography. Urbana and Chicago: University of Illinois press.

Osgood, C.E. (1952). The Nature and Measurement of Meaning. Psychological Bulletin, 49(3), 197327.

Poston, T. \& Stewart, I. (1978). Catastrophe Theory and Its Applications. London, San Francisco, Melbourne: Pitman.

Smith, M.B. 1947. The Personal Setting of Public Opinions: A Study of Attitudes Towards Russia. Public Opinion Quarterly. 11(4), 507523. 